\title{
Mouse sperm acquire a new structure on the apical hook during epididymal maturation
}

\author{
Yi-Wen Lin ${ }^{1}$, Tzu-Han Hsu ${ }^{2}$ and Pauline H Yen ${ }^{1}$
}

Spermatozoa emerging from the testis undergo a maturation process in the epididymis during which they change morphologically, biochemically and physiologically to gain motility and the ability to fertilize ova. We examined mouse epididymal sperm with immunostaining and transmission electron microscopy (EM) and identified a previously unknown structure on the apical hook. The structure has a coiled configuration around $11 \mathrm{~nm}$ in thickness and is present at the tip of each corner of the triangular-rod shaped perforatorium. Surveying sperm isolated from various regions of the epididymis indicated that mouse sperm acquire the hook rim (HR) structure during its passage through the proximal two-thirds of the caput epididymidis. The structure withstands vigorous sonication and harsh chemical treatments and remains intact after the acrosome reaction. Its location and sturdiness suggest a function in protecting the apical hook from mechanical wear during fertilization. Our EM images of epididymal sperm also revealed additional novel structures as well as lateral asymmetry of the sperm head, indicating that mouse sperm head has a structure more complex than previously recognized.

Asian Journal of Andrology (2013) 15, 523-528; doi:10.1038/aja.2013.46; published online 3 June 2013

Keywords: apical hook; epididymal maturation; mouse sperm; sperm head ultrastructure

\section{INTRODUCTION}

Both rat and mouse spermatozoa have a falciform-shaped head that is composed of four major parts: the nucleus, the acrosomic system, the perinuclear theca and the plasma membrane. ${ }^{1,2}$ The nucleus takes up most of the head volume and contains the haploid genome tightly packed with protamines. The acrosomic system consists of two distinct regions, the acrosome cap (also known as acrosome proper, anterior acrosome) and the head cap (also known as equatorial segment, posterior acrosome). The acrosome cap sits on top of the nucleus in the anterior-dorsal part of the head and contains numerous digestive enzymes that facilitate the passage of the sperm through the cumulus cell layer and the zona pellucida during fertilization. ${ }^{3}$ The head cap is much thinner and covers much of the lateral surface of the head. ${ }^{4}$ The perinuclear theca is a cytoskeletal structure that covers most of the nucleus except in the area of the perifossal zone near the base of the head where the tail is attached. It consists of the subacrosomal region (also known as the perforatorium), and the postacrosomal sheath (also known as postacrosomal dense lamina). The perforatorium in rats and mice is in the shape of a curved triangular rod at the apex of the sperm head, and splits into three interconnected prongs, one dorsal and two ventral, as it passes over the nucleus. ${ }^{5,6}$ It is thought to play a mechanical role in egg penetration during fertilization. ${ }^{1}$ The postacrosomal sheath covers the posterior portion of the nucleus and thickens to form the ventral spur along the ventral side of the nucleus. The plasma membrane wraps the surface of the head and is subdivided into various regional domains that overlay different organelles and structures and have distinct protein and lipid components. ${ }^{2}$
Although sperm are produced in the testis, the fully developed testicular spermatozoa are nonfunctional. They require a maturation process in the epididymis to acquire motility and fertilizing ability. ${ }^{7}$ The epididymis is a highly compartmentalized organ with a long tubule structure that is commonly divided into the caput, corpus and cauda sections. ${ }^{7,8}$ The various sections have distinct secretomes and proteomes and serve different functions. ${ }^{8,9}$ Sperm maturation occurs primarily in the caput epididymidis, and is accompanied with numerous morphological, biochemical and physiological changes. ${ }^{8,10}$ The cytoplasmic droplet moves along the mid-piece of the tail. The acrosome changes its shape, which is substantial in some species and subtle in others, as well as its content. ${ }^{10,11}$ The structures of several sperm organelles are modified, primarily due to disulfide crosslinking of their protein components. The sperm plasma membrane also undergoes changes in surface charge as well as lipid content and distribution. ${ }^{2,12}$ In addition, the distribution and post-translational modification of numerous proteins are also altered. ${ }^{13-15}$ Through the process, the sperm gain the ability to swim forward and the capacity to undergo acrosome reaction and fertilize an ovum. ${ }^{16}$ The mature sperm are then stored in the cauda epididymidis.

We previously generated an antibody against a synthetic oligopeptide consisting of a 19 amino acid sequence in the germ cell-specific ubiquitin-specific protein 26 (USP26). ${ }^{17}$ The antibody specifically stained cultured cells transfected with an USP26 expression vector but not the empty vector, and immunoprecipitated USP26 with deubiquitinating enzymatic activity from mouse testis lysates. However, the presence of multiple bands on Western blots hinted

${ }^{1}$ Institute of Biomedical Sciences, Academia Sinica, Taipei 11529, China and ${ }^{2}$ Institute of Cellular and Organismic Biology, Academia Sinica, Taipei 11529 , China Correspondence: Dr PH Yen (pyen@ibms.sinica.edu.tw)

Received: 30 January 2013; Revised: 22 February 2013; Accepted: 30 March 2013; Published online: 3 June 2013 
possible crossreaction of the antibody with other proteins. ${ }^{17}$ Here we report our subsequently immunostaining of mouse epididymal sperm with the antibody that led to the discovery of a novel structure we have named 'hook rim (HR)' at the three edges of the triangular rod of the anterior perforatorium. Examination of sperm from various regions of mouse epididymis revealed that sperm acquire HRs during epididymal maturation. The location and sturdiness of the HR suggest a function in protecting the apical hook from mechanical wear during fertilization. Our electron microscopy (EM) examination also uncovered lateral asymmetry and some novel structures of the mouse sperm head.

\section{MATERIALS AND METHODS}

\section{Animals}

C57BL/ 6 mice used in the study were $6-8$ weeks old. They were housed in a specific pathogen-free animal facility, and the experiments were approved by the Institutional Animal Care \& Utilization Committee of Academia Sinica (Taipei, China).

Immunostaining of tissue sections and dispersed germ cells Immunostaining of Bouin's solution-fixed, paraffin-embedded mouse testicular and epididymal sections was carried out as previously described. ${ }^{18}$ Spermatozoa were isolated from different regions of mouse epididymis according to Olson et al. ${ }^{11}$ They were fixed with $2 \%$ paraformaldehyde in phosphate-buffered saline and spun onto glass slides using the Shandon Cytospin 4 Cytocentrifuge (Thermo Scientific, Pittsburgh, PA, USA). The cells were stained with the anti-USP26-M antibody ${ }^{17}$ and an Alexa Fluor 488 conjugated goat anti-rabbit IgG secondary antibody (Invitrogen, Carlsbad, CA, USA) according to Lin and Yen. ${ }^{19}$ The acrosome was labeled with lectin peanut agglutinin (PNA) conjugated with Alexa Fluor 594 (Invitrogen) or a mouse monoclonal anti-acrosin antibody (GeneTex, Inc., Irvine, CA, USA), and the nucleus was stained with Hoechst 33258 (Invitrogen). The cells were then mounted and photographed with a Zeiss LSM510 confocal microscope (Carl Zeiss Microscopy GmbH, Jena, Germany).

\section{Immunogold staining and EM}

Transmission EM was carried out using the service of the Electron Microscope Core Facility at Academia Sinica (Taipei, China). Adult cauda epididymides were fixed in $4 \%$ paraformaldehyde, $0.1 \%$ glutaraldehyde and $0.05 \%$ tannic acid in $0.1 \mathrm{~mol} \mathrm{l}^{-1}$ cacodylate buffer ( $\mathrm{pH}$ 7.3) and embedded in epoxy resin. Thin sections prepared were stained with uranyl acetated and examined under a Hitachi H7000 transmission EM (Hitachi High-Technologies Co., Tokyo, Japan) operating at $75 \mathrm{kV}$ under standard operating conditions. Wholemount sperm specimens were prepared according to a published protocol, ${ }^{11}$ and immuno-gold staining was performed as previously described. ${ }^{17}$ Perforatorial triangular cross-sections were photographed regardless whether they contained gold-labels or not, and the distribution of the gold-labels on the images was then analyzed.

\section{Various treatments of epididymal sperm}

The sperm were treated separately with (i) six cycles of sonication on the Ultrasonic Homogenizer UP50H (Hielscher, Teltow, Germany) using the 'six times at $25 \%$ amplitude' program according to Lin et al. ${ }^{20}$ (ii) $20 \mathrm{mmol} \mathrm{l}^{-1} \beta$-mercaptoethanol in the RIPA buffer for $15 \mathrm{~min}$ at $4^{\circ} \mathrm{C}$; (iii) $1 \mathrm{~N} \mathrm{NaOH}$ at room temperature for $30 \mathrm{~min}$; (iv) $7 \mathrm{moll}^{-1}$ urea in $150 \mathrm{mmoll}^{-1} \mathrm{NaCl}$ at room temperature for an hour; and (v) $0.2 \mathrm{mg} \mathrm{ml}^{-1}$ trypsin at $37^{\circ} \mathrm{C}$ for an hour. For (i), (iv) and (v), sperm were rinsed in phosphate-buffered saline after the treatments, fixed in 4\% paraformaldehyde, spun onto glass slides and stained with the anti-USP26 antibody and Hoechst 33258. For (ii) and (iii), the fragmented sperm were spun onto glass slides before being fixed and stained.

\section{RESULTS}

Identification of a novel structure on mouse epididymal sperm We immunostained mouse spermatozoa with the anti-USP26 antibody, and labeled the acrosome with PNA which binds specifically to the acrosome outer membrane. ${ }^{21,22}$ Fully developed spermatozoa isolated from whole mouse testes showed intense USP26 signals on the dorsal surface of the head, covering most of the frontal portion of the head as previously reported ${ }^{17}$ (Figure 1a). The USP26 and PNA signals partially overlapped and both covered the apical hook. However, spermatozoa isolated from caudal epididymides gave a completely different staining pattern (Figure 1b). The USP26 signals were now restricted to the apical hook and assumed a unique three-prong structure, with a dorsal prong and two ventral prongs that were of different lengths. Conversely, the PNA signals had retracted from the apical tip and most of the lateral surface, and became confined to the dorsal ridge of the head showing no overlap with the USP26 signals. An

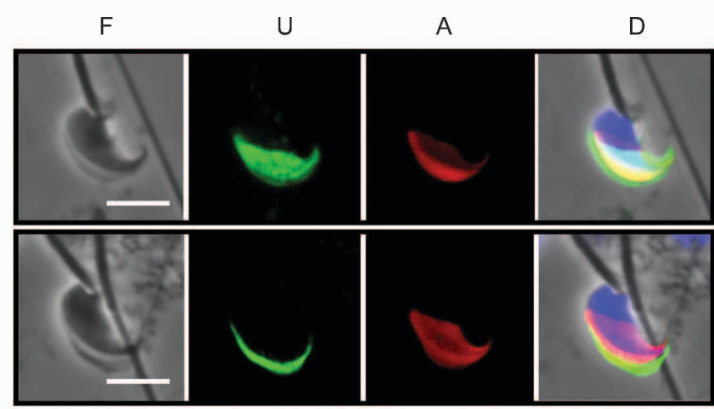

b

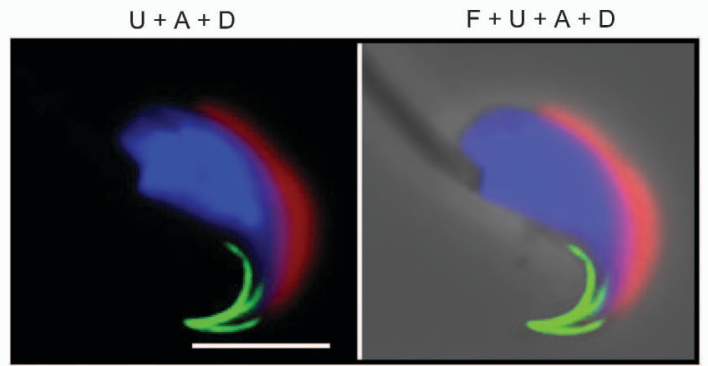

c

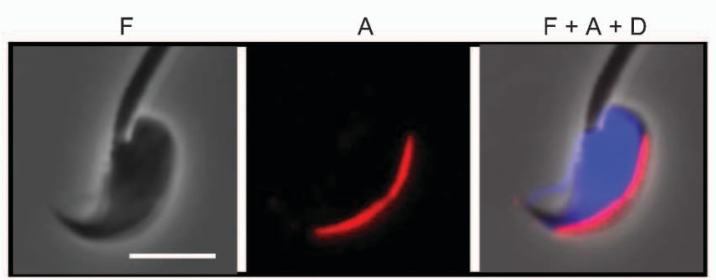

Figure 1 Remodeling of mouse sperm head during epididymal maturation. Sperm were isolated from mouse testes (a) and caudal epididymides (b, c) and stained for the USP26 epitope (U), acrosome (A) and DNA (D). Acrosomes were stained with PNA in $\mathbf{a}$ and $\mathbf{b}$, and an anti-acrosin antibody in $\mathbf{c}$. Phase-contrast (F) images of the sperm are also presented, either alone or merged with the immunofluorescence images. Scale bar $=5 \mu \mathrm{m}$. PNA, peanut agglutinin; USP26, ubiquitin-specific protein 26. 
antibody against acrosin, a soluble content of the acrosome, ${ }^{23}$ produced a similar staining pattern of the acrosome (Figure 1c).

\section{Ultrastructure of the head of mature mouse spermatozoon}

We next attempted to identify the structure detected by the antiUSP26 antibody. A literature search revealed that while the ultrastructure of the rat sperm head was well documented, based primarily on the structure of step 19 spermatid, ${ }^{1,4,24}$ the structure of a mature mouse sperm head had not been characterized in detail. ${ }^{5,25,26}$ We thus used EM on both whole-mount cauda epididymidal sperm and thin sections of cauda epididymidis to determine the ultrastructure of mouse sperm head, paying special attention to the apical hook (Figure 2). Images of the whole-mount sperm showed that the triangular rod of the anterior perforatorium was capped by a previously unnoticed circular structure of $160 \mathrm{~nm}$ in length by $40 \mathrm{~nm}$ in diameter (Figure 2a inset). Examination of cauda epididymidal sections revealed that the mouse sperm head had a structure very similar to that of the head of a rat step 19 spermatid. ${ }^{4}$ The anterior perforatorium
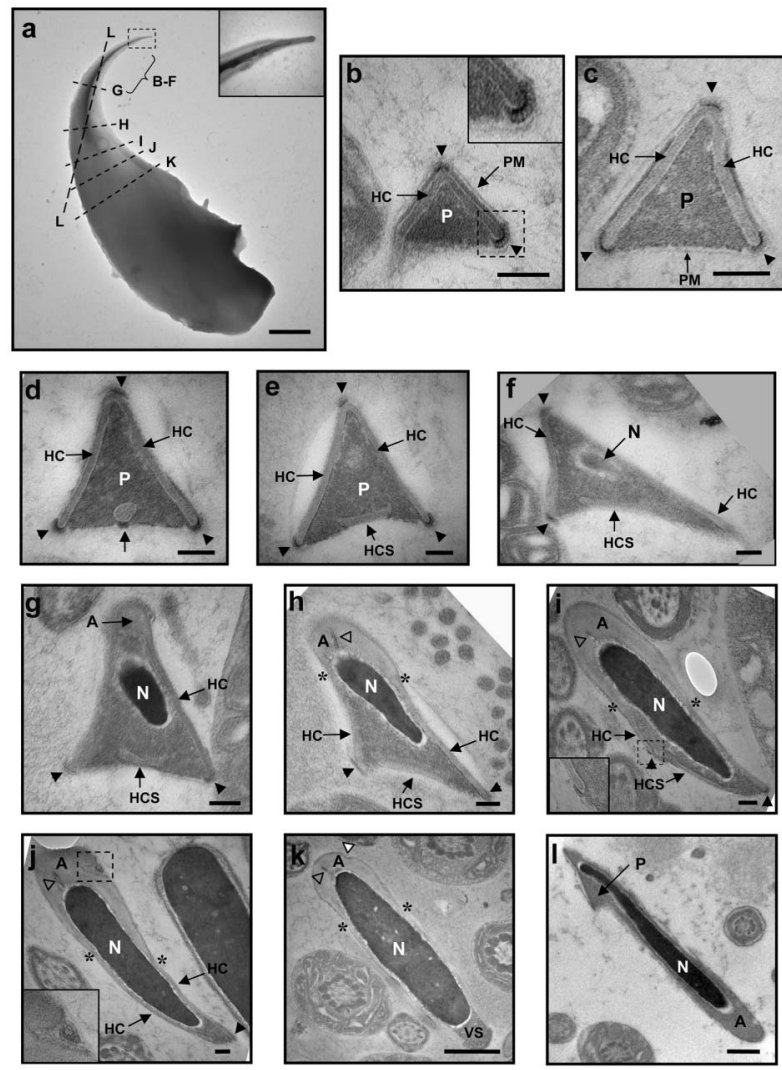

Figure 2 Electron micrographs of mouse cauda epididymidal sperm. (a) Image of a whole-mount sperm head. The bracket marks the region from where crosssections in b-f are derived, whereas the broken lines correspond to the planes of cross-sections in $\mathbf{g}-\mathbf{I}$. The cross-sections are localized on the sperm head based on their longitudinal lengths. In $\mathbf{b}$-k, the dorsal edges of the perforatorial triangles point to the top, whereas in I, the apical tip of the hook points to the top-left corner. Arrowheads in $\mathbf{b}$ - $\mathbf{j}$ point to the HRs at the apexes; asterisks in $\mathbf{h}-\mathbf{k}$ locate the limits between the acrosome cap and the head cap; open triangles in $\mathbf{h}-\mathbf{k}$ point to the indents inside the acrosome, and the white triangle in $\mathbf{k}$ points to the indent on the acrosome surface. In $\mathbf{a}, \mathbf{b}, \mathbf{i}$ and $\mathbf{j}$, the boxed regions are enlarged in the insets. For a, scale bar $=1 \mu \mathrm{m}$; for $\mathbf{b}-\mathbf{j}$, scale bar $=100 \mathrm{~nm}$; for $\mathbf{k}$ and $\mathbf{I}$, scale bar $=500 \mathrm{~nm}$. A acrosome cap; HC, head cap; HCS, separated head cap segment; N, nucleus; P, perforatorium; PM, plasma membrane; VS, ventral spur. was covered by the head cap (HC) on the top and the two lateral sides (Figure $\mathbf{2 b}-\mathbf{2 e}$ ). Cross-sections taken progressively away from the tip of the hook showed the sequential appearance of an electron-dense spot connecting to an electron-lucent circle at the center of the ventral surface (Figure 2d, indicated with an arrow) which later became the separated head cap segment (HCS) (Figure 2e), the elliptically shaped nucleus (Figure 2f) and the acrosome cap (Figure 2g). Further down the hook, one of the bottom apexes of the perforatorial triangle started to retract (Figure $\mathbf{2 h}$ and $\mathbf{2 i}$ ), the HC on one side fused with the HCS to become one continuous membranous structure (Figure $2 \mathbf{j}$ ), and the remaining perforatorial/perinuclear theca material rounded up to form the ventral spur (Figure 2k).

We noticed several features that had not been previously reported for the rat sperm head. Firstly, there were electron-dense regions (indicated with arrowheads) with a unique ultrastructure (Figure $\mathbf{2 b}$ inset) at most apexes of the perforatorial triangular cross-sections. The structure, designated HR, had a coiled configuration around $11 \mathrm{~nm}$ in thickness. At the top dorsal apex of the triangle, the HR sat on top of the HC (Figure 2b-2f), whereas at the basal ventral corners, the HRs wrapped around the ends of the HCs (Figure $\mathbf{2} \mathbf{b}-\mathbf{2 i}$ ). The top HR disappeared when the acrosome cap appeared (Figure 2g), whereas the two bottom HRs disappeared at the fusion of the HC and HCS (Figure 2j) and the formation of ventral spur (Figure 2k), respectively. Secondly, in some small perforatorial triangles, the HC on one side protruded beyond the baseline to form a tail-like structure (Figure $\mathbf{2 b}$, also Figure $\mathbf{4 b}$ left panel). Thirdly, some cross-sections of the acrosome cap showed an indent that contained a unique double-membrane round structure (Figure 2j inset). Finally, our EM examination revealed previously unrecognized lateral asymmetry of the mouse sperm head. The nuclei in the perforatorial cross-sections were not centrally positioned; their ventral ends tilted toward one of the basal apexes (Figure $2 \mathbf{f}-2 \mathbf{h}$ ). A section cutting through the dorsal portion of the apical hook clearly showed an asymmetrical odd shape of the frontal apical hook (Figure 2a and 2l).

We next performed immunogold staining of mouse cauda epididymidal sections to identify the structure on the apical hook detected by the anti-USP26 antibody. The anti-USP26 antibody labeled most perforatorial triangular cross-sections, and the vast majority of goldlabels fell within or close to the HRs at the apexes (Figure 3a and 3b). When the control antibody was used, only a small fraction of perforatorial cross-sections contained gold labels and those labels were not clustered at the HRs (Figure $\mathbf{3 c}$ and $\mathbf{3 d}$ ). Thus, the anti-USP26 antibody marked the HRs at the apexes of the perforatorial triangular rod. This conclusion is also supported by the good agreement between the extent of HRs' presence (detected by EM) and that of the USP26 immunofluorescence signals along the edges of the apical hook.

\section{Formation of the HR during epididymal maturation}

In their careful examination of the ultrastructure of rat step 19 spermatid, Lalli and Clermont ${ }^{4}$ did not note any electron-dense structures at the apexes of the triangular perforatorium, suggesting that sperm acquired the HR in the epididymis. We therefore examined epididymal sections prepared from the proximal caput where sperm first enter epididymis (Figure 4a plane a). Most cross-sections of the anterior perforatoria lacked electron-dense regions at their apexes (Figure 4b), indicating that the HRs took shape in the epididymis.

To investigate where in the epididymis sperm acquired the HRs, we divided the mouse epididymis into five regions, ${ }^{16}$ including three regions for the caput section and one region each for the corpus and cauda sections of the epididymis (Figure 4a), and examined 
a

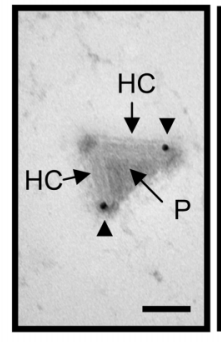

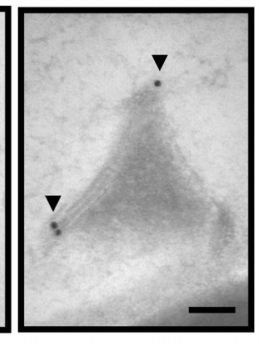
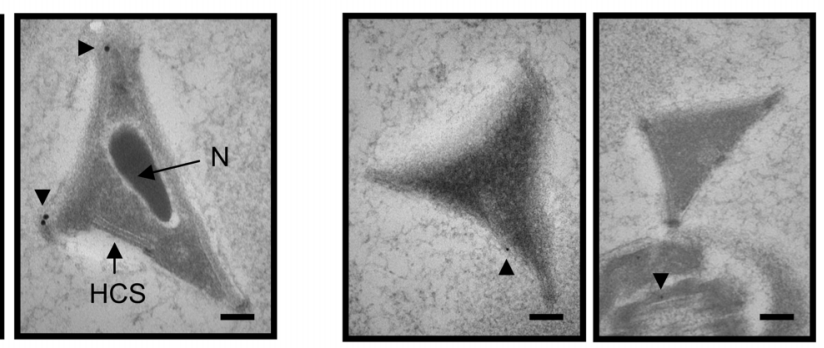

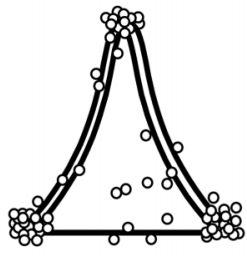

26 cross sections 67 labels

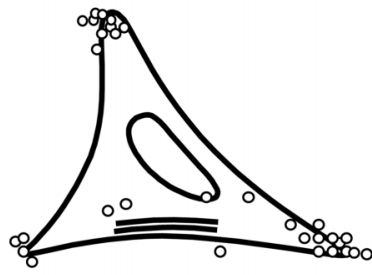

13 cross sections 28 labels d

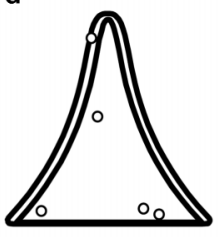

16 cross sections 5 labels

Figure 3 Immunogold labeling of the USP26 epitope on cross-sections of cauda epididymidal sperm head. (a) Examples of anterior perforatorial cross-sections with gold labels at or near the electron-dense regions at the apexes. The gold labels are $18 \mathrm{~nm}$ in diameter. (b) Distribution of gold labels (open circles) on perforatorial crosssections with (right) or without (left) nuclei. The number of cross-sections scored and total gold labels observed are indicated at the bottom. (c, d) EM images and goldlabel distribution on perforatorial cross-sections stained with the control antiserum. The gold labels are $12 \mathrm{~nm}$ in diameter. For $\mathbf{a}$ and $\mathbf{c}$, scale bar=100 nm. The arrowheads point to the gold labels. EM, electron microscopy; HC, head cap; HCS, separated head cap segment; N, nucleus; P, perforatorium.

spermatozoa isolated from each region (Figure 4c). In Region 1, most germ cells still had the PNA and USP26 signals covering a large portion of the head. However, the major ventral prong (indicated by arrows) started to take shape at the apical tip, well separated from the main body of the USP26 crescent. For germ cells in Region 2, both the PNA and the USP26 signals had contracted to a crescent shape at the dorsal ridge of the head. The frontal half of the major ventral prong thickened, and the dorsal and the minor ventral prongs started to bud out from the frontal tip and midpoint of the major ventral prong, respectively (inset). Spermatozoa in Region 3 showed the same PNA and USP26 staining patterns as those of the cauda epididymidal sperm described above, with the USP26 signals confined to the now completed three-prong structure at the apical hook. Spermatozoa isolated from regions 4 and 5 had the same staining patterns as those from Region 3 (data not shown). The EM and immunostaining results indicated that the sperm acquired HRs in the proximal two-thirds of the caput epididymidis.

\section{Characterization of the HR}

We further studied the effects of various treatments on the HR by following the USP26 immunofluorescence signals (Figure 5). Staining caudal epididymidal sperm with the pre-immune serum (Figure 5a) or the anti-USP26 antibody preincubated with the oligopeptide immunogen (Figure 5c) produced no signals at the HRs. Subjecting the sperm to repeated sonication, a condition that disrupts the outer membrane of acrosome and releases its soluble content, ${ }^{20}$ had little effect on the three-prong structure (Figure 5d). Induction of acrosome reaction with the calcium ionophore A23187 significantly reduced the signals associated with the two minor prongs but not those associated with the major ventral prong (Figure 5e). It was previously reported that the perforatorium resisted solubilization by several harsh treatments, including $1 \%$ SDS, $1 \%$ sarkosyl, 4 mol $1^{-1}$ urea or $6 \mathrm{~mol} \mathrm{l}^{-1}$ urea in the presence of $2 \mathrm{mmol} \mathrm{l}^{-1} \mathrm{DTT},{ }^{27}$ or $1 \mathrm{~N}$ $\mathrm{NaOH},{ }^{6}$ likely due to extensive disulfide linking of its protein components. ${ }^{28}$ In our hand, the three-prong HR structure also survived $20 \mathrm{mmol} \mathrm{l}^{-1} \beta$-mercaptoethanol (Figure 5f) or $1 \mathrm{~N} \mathrm{NaOH}$ (data not shown) treatment which disrupts the nucleus, and $7 \mathrm{~mol}^{-1}$ urea (Figure 5g) which dissolves most sperm proteins. However, 1-hour trypsin treatment $\left(0.2 \mathrm{mg} \mathrm{ml}^{-1}\right.$ at $\left.37^{\circ} \mathrm{C}\right)$ destroyed the lysine-rich epitope detected by the antibody (Figure $5 \mathbf{h}$ ), yet the overall structure of the apical hook remained intact (Figure 5i). Thus, the HR appears to bind tightly to the main body of the perforatorium and withstands mechanical and chemical insults.

\section{DISCUSSION}

We have identified a novel HR structure which the mouse sperm acquire during epididymal maturation. To the best of our knowledge, the HR has not been described before, though electron-dense regions were noted at the apexes of some published EM images of mouse perforatorial cross-sections. ${ }^{5}$ The HR is located at the tip of the three corners of the mouse apical hook, and is quite sturdy, not disrupted by repeated sonication, acrosome reaction or harsh chemical conditions. During fertilization, the sperm have to drill through layers of cumulus cells and zona pellucida in order to reach and penetrate the egg. The $\mathrm{HR}$ is likely to serve a function in protecting the hook from mechanical wear. The coiled structure of the HR resembles that of microtubules in a flagellum, ${ }^{29}$ and we observed weak signals at the apical hook with an anti- $\alpha$-tubulin antibody after the intensely stained sperm tails were removed by repeated sonication and differential centrifugation (Supplementary Figure 1). Antibodies against other cytoskeletal proteins such as $\beta$-actin and cytokeratins (including cytokeratin $4,5,6,8$, 10,13 and 18), or phalloidin that specifically stains F-actin, failed to 

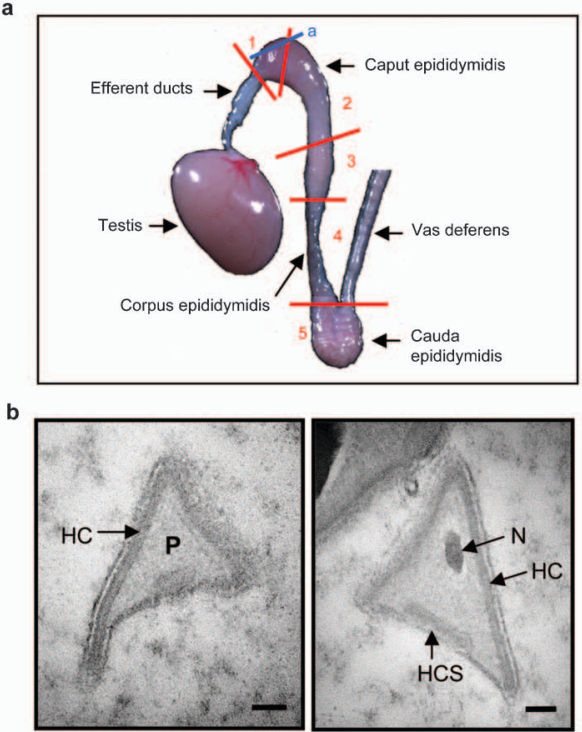

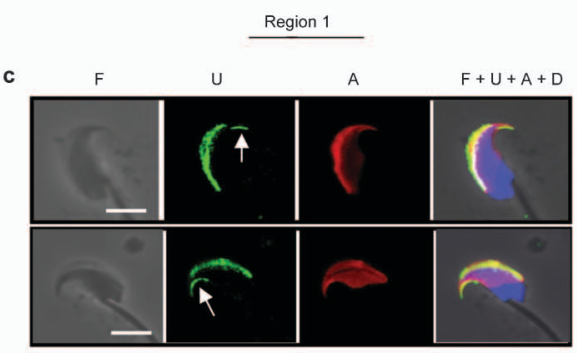

Region 2
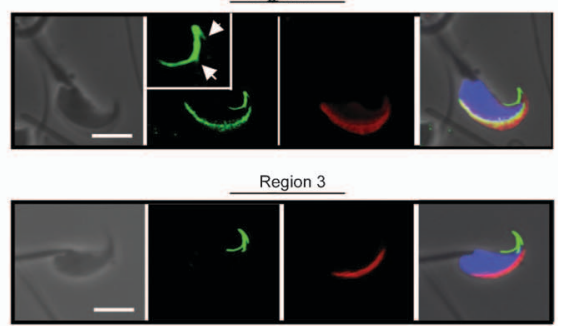

Figure 4 Formation of the HR in the caput epididymidis. (a) Mouse testis and epididymis. The red lines indicate the cuts that separate the epididymis into five different regions, and the blue line indicates the plane where thin sections for EM (in b) were prepared. (b) EM images of perforatorial cross-sections of sperm at the proximal end of caput epididymidis. Scale bar $=100 \mathrm{~nm}$. (c) Sperm isolated from various regions of the epididymis were stained for the USP26 epitope (U), acrosome (A) and DNA (D). For the sperm in Region 1, the arrows point to the primitive major ventral prong of the HR structure. For the spermatozoon in Region 2 , the HR structure in the process of formation is enlarged in the inset and the arrows point to the budding minor prongs. Scale bar $=5 \mu \mathrm{m}$. EM, electron microscopy; HC, head cap; HCS, separated head cap segment; HR, hook rim; N, nucleus; P, perforatorium; USP26, ubiquitin-specific protein 26.

label the HR (Supplementary Figure 1). Thus, the HR has a composition distinguished from that of the acroplaxome which is an F-actinkeratin-containing plate closely associated with the leading edge of the acrosome during the shaping of spermatid head. ${ }^{30}$

The HR decorates the edges of the perforatorial triangular rod. The perinuclear theca/perforatoria of rat and mouse sperm have been isolated and characterized. ${ }^{5,6,27,31}$ Both of them are composed of a major 15/16-kDa protein, designated PERF15 and several minor proteins. The cloning of rat and mouse Perf 15 cDNAs provided the protein sequences that identified PERF15 as a member of the fatty acid-binding protein (FABP) family. ${ }^{5,31}$ Mice lacking PERF15/FABP9 exhibit only minor spermatogenic defects. ${ }^{32}$ They have a significant but modest increase in sperm with head abnormalities, including misshaped apical parts and the absence of ventral spur, but the total sperm count and fertility of the mice remained normal. Antibodies raised against the whole perforatorial fraction labeled the entire perforatorium as well as the ventral spur, ${ }^{1,6}$ whereas an affinity-purified anti-PERF15 antibody specifically labeled the perforatorium. ${ }^{5,31}$ No antibody labeled the apexes of the triangular anterior perforatorium where HRs are located.

Our EM examination showed that despite a significant difference in the overall shape, the mouse epididymal sperm head has an ultrastructure very similar to that of the head component of rat step 19 spermatid, ${ }^{4}$ the only stage when detailed ultrastructure is available. However, the two rodent sperm heads differ significantly in the degree of lateral asymmetry. Lalli and Clermont ${ }^{4}$ noted only asymmetry in the locations of the limits between the acrosome cap and the head cap on rat sperm head. In mouse sperm head, we found additional asymmetry in the apical hook as revealed by the off-center location of the nucleus, the existence of a major and a minor ventral prong labeled by the anti-USP26 antibody, the presence of a double-membrane round body on one lateral side of the acrosome and the overhang of $\mathrm{HC}$ on one side of the perforatorial triangles. However, we cannot rule out the possibility that rat sperm head acquires additional asymmetry as well as the HRs during epididymal maturation. The anti-USP26 antibody failed to label the apical hook of rat epididymal sperm.

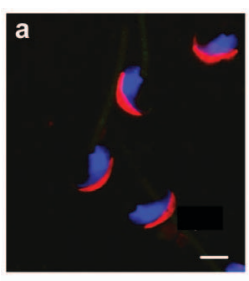

Pre-immune

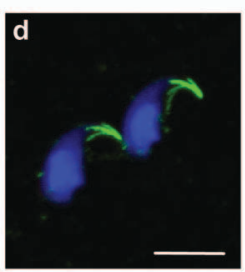

Sonication

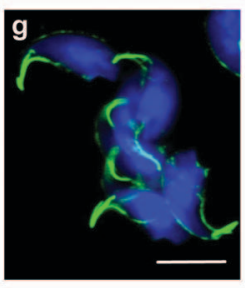

$7 \mathrm{~mol} \mathrm{I}^{-1}$ urea

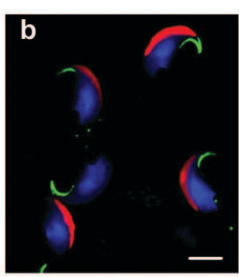

Anti-USP26

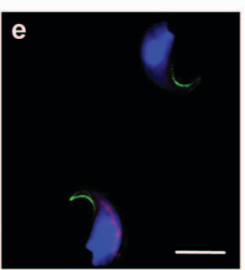

Acrosome Reaction

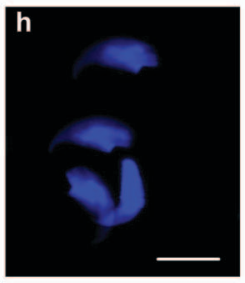

Trypsin

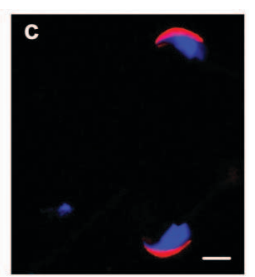

Ab+immunogen

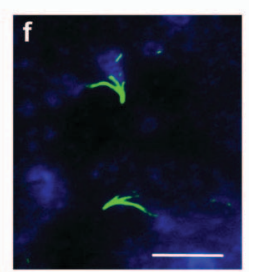

$20 \mathrm{mmol} \mathrm{I}^{-1} \beta-\mathrm{ME}$

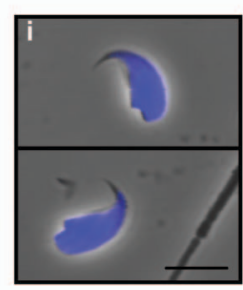

Trypsin
Figure 5 Effects of various treatments on the HRs. (a-c) Mouse cauda epididymidal sperm were stained with the pre-immune antiserum (a), the anti-USP26 antibody (b) or the anti-USP26 antibody preincubated with the immunogen (c). Positive antibody binding is indicated by green signals. The cells were also stained for the acrosome (red) and nucleus (blue). (d-i) The sperm were subjected to the various treatments as indicated and stained for the USP26 epitope (green), acrosome (red) and nucleus (blue) afterward. In i, phase-contrast images of trypsin-treated sperm are superimposed with the fluorescence images. Scale bar $=5 \mu \mathrm{m}$. HR, hook rim; USP26, ubiquitin-specific protein 26. 
The identity of the protein at the HR detected by the anti-USP26 antibody remains undetermined. It could be USP26 per se since the ubiquitin-proteasome system has been shown to be involved in the fertilization of several mammalians. ${ }^{33}$ USP26 is predicted to be a cytosolic protein based on sequence alignments and the three-dimensional structures of other USP proteins. ${ }^{34,35}$ However, USP26 consists of several segments/insertions that are absent from other members of the USP family, and the lysine-rich segment (DKKAKPTRKVDPTKFNKKE) used to generate the anti-USP26 antibody is present in one of the insertions. The segment was selected many years ago for lack of significant homology with other mouse proteins in the databases. However, a recent database search identified several mouse proteins with identical hexapeptide sequences or up to $53 \%$ of scattered identities. Preincubation of the anti-USP26 antibody with oligopeptides covering different portion of the immunogen (DKKAKPTRK, RKVDPTKL and KLNKKE) failed to compete out the signals at the HRs, suggesting that the antiUSP26 antibody either recognizes scattered amino-acid identity or three-dimensional structure of an unknown protein, or a processed USP26 at the HR. The epitope is present on the outside surface of the plasma membrane since direct staining of the sperm without prior permeabilization and fixation generated the same staining pattern (data not shown). The protein with the epitope, designated X, is likely a transmembrane protein with its cytoplasmic domain attached to the electron-dense HR structure underneath the plasma membrane. We failed in several attempts to determine the identity of the $\mathrm{X}$ protein, including purification of mouse perforatoria followed by mass spectrometry, due to the problem of insolubility.

In summary, our immunostaining and EM examination of mouse epididymal sperm have revealed new details in the ultrastructure of mouse sperm head, including lateral asymmetry of the head and the HR structure which the sperm acquire during epididymal maturation.

\section{AUTHOR CONTRIBUTIONS}

YWL designed and carried out most of the experiments and drafted the manuscript. THH performed the EM experiments. PHY participated in the design of the experiments and finalized the manuscript. All authors read and approved the final manuscript.

\section{COMPETING FINANCIAL INTERESTS}

The authors declare no competing financial interests

\section{ACKNOWLEDGMENTS}

We thank Anli Huang for her assistance with the EM, and Ching-Shu Suen and Santai Shen for analyzing the 3D structure of USP26. The work was supported by a postdoctoral fellowship to YWL and the intramural funding from Academia Sinica.

Supplementary Information accompanies the paper on Asian Journal of Andrology's website (http://www.nature.com/aja).

1 Clermont Y, Oko R, Hermo L. Cell biology of mammalian spermiogenesis. In: Desjardin C, Ewing LL, editors. Cell and Molecular Biology of the Testis. New York: Oxford University Press; 1993. pp332-76.

2 Eddy E. The spermatozoon. In: Neill JD, editor. Knobil and Neill's Physiology of Reproduction. 3rd ed. New York: Elsevier; 2006. pp3-54.

3 Jin M, Fujiwara E, Kakiuchi $Y$, Okabe M, Satouh $Y$ et al. Most fertilizing mouse spermatozoa begin their acrosome reaction before contact with the zona pellucida during in vitro fertilization. Proc Natl Acad Sci USA 2011; 108: 4892-6.

4 Lalli M, Clermont $Y$. Structural changes of the head components of the rat spermatid during late spermiogenesis. Am J Anat 1981; 160: 419-34.

5 Korley R, Pouresmaeili F, Oko R. Analysis of the protein composition of the mouse sperm perinuclear theca and characterization of its major protein constituent. Biol Reprod 1997; 57: 1426-32.
6 Oko R, Clermont Y. Isolation, structure and protein composition of the perforatorium of rat spermatozoa. Biol Reprod 1988; 39: 673-87.

7 Robaire B, Hinton B, Orgebin-Crist M. The epididymis. In: Neill JD, editor. Knobil and Neill's Physiology of Reproduction. 3rd edn. New York: Elsevier; 2006. pp1071-148.

8 Cornwall GA. New insights into epididymal biology and function. Hum Reprod Update 2009; 15: 213-27.

9 Dacheux JL, Belleannee C, Jones R, Labas V, Belghazi M et al. Mammalian epididymal proteome. Mol Cell Endocrinol 2009; 306: 45-50.

10 Bedford JM. Enigmas of mammalian gamete form and function. Biol Rev Camb Philos Soc 2004; 79: 429-60.

11 Olson GE, Winfrey VP, Nagdas SK. Structural modification of the hamster sperm acrosome during posttesticular development in the epididymis. Microsc Res Tech 2003; 61: 46-55

12 Jones R, James PS, Howes L, Bruckbauer A, Klenerman D. Supramolecular organization of the sperm plasma membrane during maturation and capacitation. Asian J Androl 2007; 9: 438-44.

13 Baker MA, Witherdin R, Hetherington L, Cunningham-Smith K, Aitken RJ. Identification of post-translational modifications that occur during sperm maturation using difference in two-dimensional gel electrophoresis. Proteomics 2005; 5: 1003-12.

14 Hunnicutt GR, Koppel DE, Myles DG. Analysis of the process of localization of fertilin to the sperm posterior head plasma membrane domain during sperm maturation in the epididymis. Dev Biol 1997; 191: 146-59.

15 Phillips DM, Jones R, Shalgi R. Alterations in distribution of surface and intracellular antigens during epididymal maturation of rat spermatozoa. Mol Reprod Dev 1991; 29: 347-56.

16 Yeung C, Cooper T. Acquisition and development of sperm motility upon maturation in the epididymis. In: Robaire B, Hinton BT, editors. The Epididymis: From Molecules to Clinical Practice. New York: Kluwer Academic/Plenum Publishers; 2002. pp417-34.

17 Lin YW, Hsu TH, Yen PH. Localization of ubiquitin specific protease 26 at blood-testis barrier and near Sertoli cell-germ cell interface in mouse testes. Int J Androl 2011; 34: e368-77.

18 Huang WJ, Lin YW, Hsiao KN, Eilber KS, Salido EC et al. Restricted expression of the human DAZ protein in premeiotic germ cells. Hum Reprod 2008; 23: 1280-9.

19 Lin YT, Yen PH. A novel nucleocytoplasmic shuttling sequence of DAZAP1, a testisabundant RNA-binding protein. RNA 2006; 12: 1486-93.

20 Lin YN, Roy A, Yan W, Burns KH, Matzuk MM. Loss of zona pellucida binding proteins in the acrosomal matrix disrupts acrosome biogenesis and sperm morphogenesis. $\mathrm{Mol}$ Cell Biol 2007; 27: 6794-805.

21 Cheng FP, Fazeli A, Voorhout WF, Marks A, Bevers MM et al. Use of peanut agglutinin to assess the acrosomal status and the zona pellucida-induced acrosome reaction in stallion spermatozoa. J Androl 1996; 17: 674-82.

22 Mortimer D, Curtis EF, Miller RG. Specific labelling by peanut agglutinin of the outer acrosomal membrane of the human spermatozoon. J Reprod Fertil 1987; 81: 127-35.

23 Polakoski KL, Zaneveld LJ, Williams WL. Purification of a proteolytic enzyme from rabbit acrosomes. Biol Reprod 1972; 6: 23-9.

24 Hermo L, Pelletier RM, Cyr DG, Smith CE. Surfing the wave, cycle, life history, and genes/ proteins expressed by testicular germ cells. Part 1: background to spermatogenesis, spermatogonia, and spermatocytes. Microsc Res Tech 2010; 73: 241-78.

25 Foster JA, Friday BB, Maulit MT, Blobel C, Winfrey VP et al. AM67, a secretory component of the guinea pig sperm acrosomal matrix, is related to mouse sperm protein sp56 and the complement component 4-binding proteins. J Biol Chem 1997; 272: 12714-22.

26 Nishizono H, Shioda M, Takeo T, Irie T, Nakagata N. Decrease of fertilizing ability of mouse spermatozoa after freezing and thawing is related to cellular injury. Biol Reprod 2004; 71: 973-8.

27 Olson GE, Hamilton DW, Fawcett DW. Isolation and characterization of the perforatorium of rat spermatozoa. J Reprod Fertil 1976; 47: 293-7.

28 Bedford JM, Calvin HI. The occurrence and possible functional significance of $-\mathrm{S}-\mathrm{S}$ - crosslinks in sperm heads, with particular reference to eutherian mammals. J Exp Zool 1974; 188: 137-55.

29 Fujiwara K, Tilney LG. Substructural analysis of the microtubule and its polymorphic forms. Ann NY Acad Sci 1975; 253: 27-50.

30 Kierszenbaum AL, Rivkin E, Tres LL. Acroplaxome, an F-actin-keratin-containing plate, anchors the acrosome to the nucleus during shaping of the spermatid head. Mol Biol Cell 2003; 14: 4628-40.

31 Oko R, Morales CR. A novel testicular protein, with sequence similarities to a family of lipid binding proteins, is a major component of the rat sperm perinuclear theca. Dev Biol 1994; 166: 235-45.

32 Selvaraj V, Asano A, Page JL, Nelson JL, Kothapalli KS et al. Mice lacking FABP9/ PERF15 develop sperm head abnormalities but are fertile. Dev Biol 2010; 348: 177-89.

33 Zimmerman S, Sutovsky P. The sperm proteasome during sperm capacitation and fertilization. J Reprod Immunol 2009; 83: 19-25.

34 Avvakumov GV, Walker JR, Xue S, Finerty PJ Jr, Mackenzie F et al. Amino-terminal dimerization, NRDP1-rhodanese interaction, and inhibited catalytic domain conformation of the ubiquitin-specific protease 8 (USP8). J Biol Chem 2006; 281: 38061-70.

35 Luna-Vargas MP, Faesen AC, van Dijk WJ, Rape M, Fish A et al. Ubiquitin-specific protease 4 is inhibited by its ubiquitin-like domain. EMBO Rep 2011; 12: 365-72. 